\title{
Combining Multiple Modes of Information using Unsupervised Neural Classifiers
}

\author{
Khurshid Ahmad ${ }^{1}$, Matthew Casey ${ }^{1}$, Bogdan Vrusias ${ }^{1}$ and Panagiotis Saragiotis ${ }^{1}$ \\ ${ }^{1}$ Neural Computing Group, Department of Computing, School of Electronics and Physical Sci- \\ ences, \\ University of Surrey, Guildford, Surrey, GU2 7XH, UK \\ $\{$ K.Ahmad, M.Casey, B.Vrusias, P.Saragiotis\}@surrey.ac.uk \\ http://www.computing.surrey.ac.uk/
}

\begin{abstract}
A modular neural network-based system is presented where the component networks learn together to classify a set of complex input patterns. Each pattern comprises two vectors: a primary vector and a collateral vector. Examples of such patterns include annotated images and magnitudes with articulated numerical labels. Our modular system is trained using an unsupervised learning algorithm. One component learns to classify the patterns using the primary vectors and another classifies the same patterns using the collateral vectors. The third combiner network correlates the primary with the collateral. The primary and collateral vectors are mapped on a Kohonen self-organising feature map (SOM), with the combiner based on a variant of Hebbian networks. The classification results appear encouraging in our attempts to classify a set of scene-of-crime images and in our attempts to investigate how pre-school infants relate magnitude to articulated numerical quantities. Certain features of SOM's, namely the topological neighbourhoods of specific nodes, allow for one to many mappings between the primary and collateral maps, hence establishing a broader association between the two vectors when compared with the association due to synchrony in a conventional Hebbian association.
\end{abstract}

\section{Introduction}

There are a number of problems currently advocated in the image understanding / retrieval literature and in the developmental psychology literature, which will benefit from the use of a system comprising a set of classifiers, capable of learning key features in the input, and using a decision combination function that can learn to combine the output of the different classifiers.

\subsection{Image Recognition and Multiple Classifiers}

Image understanding and retrieval systems, especially those discussed under the rubric of content-based image retrieval (CBIR) systems, focus on how a system can identify the components of an image by dealing, almost exclusively, on physical and/or per- 
ceptual features of the image. Typical multiple classifier ensembles used so far comprise individual 'expert' classifiers that can deal with one physical feature at a time: colour, texture, shape or illumination. The CBIR literature is increasingly using image external features, for instance, texts collateral to the image - a caption, or a news story in which the image is embedded. Schettini et al [15] have used the CART methodology to construct tree classifiers, which in turn use a majority voting rule, for classifying a set of indoor, outdoor and close up images.

In an oft-cited paper, Kittler et al have presented 'a common theoretical framework for combining classifiers which use distinct pattern representation' ([10] p.226). The framework uses the Bayesian relationship between the posterior and prior probability density functions that model one of the many possible classes, to the measurement vectors used by a given classifier. What is of interest to us here is the use of multiple classifiers that deal with perceptually (quasi-) independent biometric sensing modalities, such as frontal facial features, face profile features and (characteristic) voice features, and a combination function that is expected to lead to the establishment of personal identity. Each classifier matches an input with a stored template and an identity is produced. In a handwriting recognition experiment, Kittler et al use four different classifiers operating on the same input, with the sum rule again achieving one of the best combining schemes. Here, the authors have used a large feed forward neural network as one of the classifiers.

Jing and Zhang [8] have used genetic algorithms for evaluating the 'correctness of the result from [...] combined classifiers' (p. 486) in dealing with recognition of faces. The authors use four classifiers dealing with sub-images based on low frequency filtered images and three orientation sub-images: horizontal, vertical and diagonal. A genetic algorithm is used for determining the weights for combining the output from the classifiers. The authors show that individual classifiers, except for low frequency filtered sub-image classifiers, perform poorly when compared to a majority voting method combined classifier - and the results of a generic-algorithm (GA) weighted classifier shows a $96 \%$ recognition rate.

The use of neural networks in the classification of complex images, for instance, human faces, is limited to less than 20 classes. There are exceptions to such a limited approach: Lawrence et al [12] have developed a multiple neural network system comprising a Kohonen self-organising feature map (SOM) [11], for quantizing a set of input image samples into a topological space, and a backpropagation network, also called a convolution network, that learns to incorporate constraints that allow it to deal with an image in an invariant manner. Such multiple neural network systems have been termed multi-net systems by Sharkey [16], but there are some nuances added to concept of multi-nets [17].

A backpropagation network is in itself multi-layered (planes in parallel) and capable of detecting multiple features in an image. Lawrence et al have compared the performance of their multi-net system with another combined classifier comprising a principal component analysis (PCA) system and multi-layer perceptron (MLP). The convolutional approach outperforms the MLP in the classification of 400 images of 40 individuals. The performance of the MLP, containing up to 200 hidden nodes, is not surprising. The interesting thing for us is that this multi-net system, convolutional plus Kohonen, with substantial $a b$ initio unsupervised learning capability performs well in the difficult task of face recognition. 


\subsection{Combining Unsupervised Classifiers}

In a recent paper on the limitations on research in multiple classifier systems, Sharkey [17] suggests that this may be due to a lack of 'awareness of the range of modular designs that could be employed' (p. 108). Sharkey advocates a modular approach and illustrates the advantages with a system of classifiers that only provides a partial solution to the classification task and the combination of the partials providing the full solution. She uses a number of neural networks, multi-layer perceptrons (MLP), organised in different topologies as unitary classifiers of fault patterns in diesel engines, and compares these with modular and ensemble approaches. An approximately $2 \%$ improvement in performance is achieved with both modular and ensemble systems over the unity solutions, not quite advocating the use of modular and ensemble systems. However, intuitively we believe that modular networks should outperform ensemble networks.

In this paper, we describe a modular co-operative network, where the component networks learn through an unsupervised process, in contrast to other supervised approaches, such as Sharkey's. The unsupervised learning regimen is used to train networks that classify the input patterns and a combiner network that produces the output.

Willshaw \& von der Malsburg [20] developed a model that applied Hebbian learning [6] between a two-dimensional pre-synaptic (input) layer and a post-synaptic (output) layer of neurons to form a topological map of activations; Hebbian learning is based on the postulate that to affect learning in connections (synapses) between two neuronal cells, the strengths of the connections are increased when both sides of the connection are active. This associative learning pre-empts the use of a teacher. The production of a topological map as a consequence of a learning rule can be regarded as a rule that maximises the average mutual information between input and output signals. Kohonen's [11] SOM is based upon a similar principle to Willshaw \& von der Malsburg's, producing a statistical approximation of the input space by mapping the input to a two-dimensional output layer. The approximation is achieved by selection of features (or prototypes) that characterise the data, which are output in a topologically ordered map.

In this paper we discuss how a multi-net system can learn to classify a set of complex input patterns; the complexity lies in the fact that the input comprises a number of independent components. Each component has different information about a given input pattern. Consider, for example the case of an annotated image - an image plus its collateral description. The image features may include a texture vector together with a textual description. The texture vector informs a system about the idiosyncratic visual features of an image independently of the description and in a different manner. Collateral information helps to sharpen the query to an image database [19].

Our combination of neural networks is so organised that one network each can learn to classify an input pattern based on the sole knowledge of one component only, in contrast to other such schemes such as the so-called mixture-of-experts (ME) network [7] in which a number of networks are trained on the same set of input patterns. The behaviour of the individual networks, taken two at a time, is learnt by another network which, in turn, learns to associate classes in the individual network that are active at a given time. This synchronous activity is used to interrelate two independ- 
ent components and is learnt through association whilst the individual classifiers learn.

The co-operative multi-net architecture that we propose extends both Willshaw \& von der Malsburg [20] paradigm and Kohonen's [11] formulation, this time by connecting two SOMs together with a Hebbian network. A multi-net system that uses a Hebbian network to interrelate the output of two (or more) SOMs, can exploit some or all of the properties of Hebbian synapses. The properties of time dependence, local modification, interaction between pre- and post-synaptic signals and their correlation provides justification for the combination of independent modalities of information (images and collateral text or numbers and articulation). When combining two SOMs, the clustering of activation in either map relates an input signal to similar prototype inputs. This local information on either side of the Hebbian connection allows local modification of signals to be achieved through corresponding interaction at pre-set time steps. Furthermore, since inputs to both SOMs are related during training, the Hebbian connection modification provides correlation between the two modalities, which can be exploited during recall. These properties enable information modalities to be combined effectively to improve classification of signals through multiple, distinct classifiers, allowing the translation of classification from one modality to another.

\section{Self-organised Combinations}

A SOM uses an algorithm that transforms a continuous $n$-dimensional input signal onto a one- or two-dimensional discrete space of neurons. For each input pattern, the weight vectors in the SOM that connect the input layer to the output layer, attempt to relate to the input by computing respective values of a discriminant function. The weight vector closest to the input is deemed the winner and represents the non-linear transformation of the input onto the output. The process of competition complete, the co-operative phase takes over with the winning neuron determining the spatial location of a set of (less) excited neurons accompanying the highly excited winner. The winner's 'halo', visually created by a Gaussian neighbourhood function, displays cooperation. The topological neighbourhood is of considerable import to our method.

The input vectors for the cases we discuss comprise two vectors - a primary vector and a collateral vector; for example, an annotated image's primary vector may comprise the visual features of colour, texture and shape, and the collateral vector holds indications of the presence or absence of keywords in the image's collateral text. We train two SOMs: one for classifying input patterns based entirely on the basis of the primary vectors, one on the collateral vectors (Fig 1). Here, the primary vector is presented to the primary SOM, which is then trained on the input using Kohonen's weight update rule [11], using a Gaussian neighbourhood function, an exponentially decreasing learning rate and neighbourhood radius. Similarly, the collateral vector is presented to the collateral SOM, which in turn is trained on the input. 


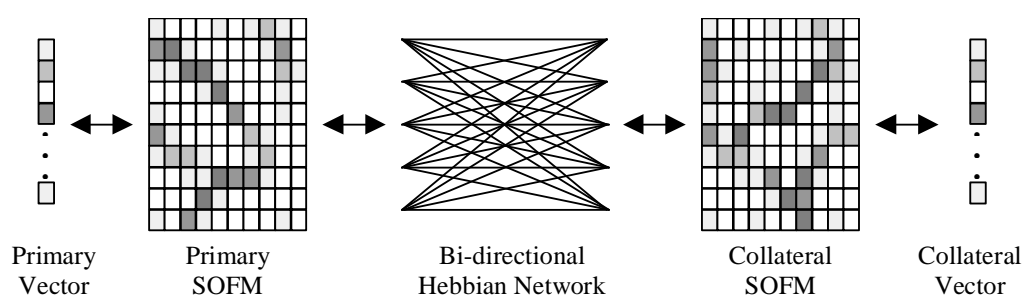

Fig 1: Architecture showing the connections between the primary and collateral SOMs. Note that training is performed in-situ with association between neighbourhoods.

Subsequent to this, the output of both the primary and collateral SOMs are input to the Hebbian network. In order to preserve the topological information provided by both SOMs, the output from each is represented as a range of activations centred on the winning neuron, demonstrating the active neighbourhood of the winner and allowing a cluster of activity to be presented to the Hebbian network. This is achieved by using the Euclidean distance for each unit in the SOM for an input, and inverting this via the function $f(x)=e^{-x}$, which provides a sufficient radius around the winner. With both primary and collateral SOM output represented in this way, the Hebbian connections were allowed to train on both of these patterns, with the cycle continuing for subsequent input patterns until sufficient learning has occurred in the SOMs to form clusters.

Each SOM shows the effects of the competition and co-operation processes used in training by assigning each pattern's neuron in the output layer. Each winning neuron has a topological neighbourhood of active neurons. The neighbours may or may not be associated with other patterns. Therefore, if during the testing phase, the presentation of a test pattern leads to its recognition by the excitation of a neuron that has won similar or the same pattern during training, the neighbourhood will also be activated.

Recall that we link the primary and collateral vectors through Hebbian learning. During the training phase, the presentation of the two vectors comprising a pattern trivially leads to a weighted one-to-one association simply because both patterns are present at the same time. However, in addition to this association by synchrony, the winning unit of a primary vector gets associated not only with the winning unit of the collateral (and vice versa), but also the neighbourhood of the winner gets associated with that of the neighbourhood of the winning unit of the collateral (and vice versa).

\subsection{Classifying Images and Collateral Text}

We first look at the combination of image and text classifiers, the image forming the primary vector, and the text associated with the image the collateral vector, taken from the scene-of-crime domain. An image is represented by a 112-dimensional vector that consists of extracted physical features. The intention when extracting these $112 \mathrm{com}-$ ponents was to create vectors that described various properties such as colours, edges and textures. In these vectors we used 21 dimensions to describe the colour distribution as an intensity histogram, 19 dimensions to describe edges by applying an edge filter and the water-filling algorithm [21], and 72 dimensions to describe texture using 
Grey Level Co-occurrence Matrices [5]. Here, the primary 15 by 15 unit SOM is intended to organise the images into clusters that share similar image features.

Collateral text is represented by a 50-dimensional (term based) binary vector. These are generated by extracting significant terms from the text, ignoring punctuation, numerical expressions and closed-class words. These significant terms are generated using the frequency of a term and a weirdness coefficient describing the subject-specificity of a term [2]. Textual vectors with common key-terms are clustered together in a 15 by 15 unit SOM.

To evaluate the proposed architecture, we trained three separate systems to allow for a comparison of results: the combined architecture, two separate SOMs, one each for images and texts, and a single SOM trained on combined image and text vectors. A total of 66 images and associated texts where used, pre-classified into 8 ideal classes by experts on the scene-of-crime domain: 'bar area', 'exhibits', 'fingerprints', 'footmarks', 'fruit machine area', 'body', 'window area' and 'general'. One vector was then selected at random from each class for use in testing and the remaining 58 were used for training.

Each system was trained for 1000 epochs with training vectors, with initial random weights, Gaussian neighbourhood function with initial radius 8 , reducing to 1 , and exponential learning rate starting at 0.9 reducing to 0.1 . The Hebbian weight connections were normalised and a learning rate of 10 was used. For the combined system, we first tested the performance of the Hebbian network on the training data by translating one SOM's output to the other SOM (image to text, text to image). We calculated the Euclidean distance of the actual SOM's winning node for a particular input, to the node that the Hebbian link activated. The results showed that the Hebbian network managed to identify all images correctly for a given textual input, and only missed 1 out of 58 texts for a given image input.

The system was then tested for its accuracy on classifying each of the 8 test inputs. Here, of the 8 test vectors, the image SOM correctly classified 4. For the remaining misclassified vectors, the collateral text vectors were input to the text SOM, and subsequently provided activation through the Hebbian link to obtain an image classification. This technique correctly classified 3 of the remaining 4 vectors. A similar approach was applied to the text SOM, giving 5 initially correct classifications, and 2 more via the image SOM and Hebbian linkage.

In comparison, the independently trained SOMs were tested with the same test vectors. The image SOM showed correct classification of 4 out of 8 test vectors, the text SOM 5 out of 8 . The combined system therefore shows the benefit of combining modalities of information to improve classification, allowing an improved response by selecting the best possible via the Hebbian connection. However, the multi-net approach shows benefit over a monolithic solution, as demonstrated by the single SOM that was trained with the input vectors formed by concatenating the image and text vectors together. Test results show worse classification ability, with only 3 out of 8 correctly classified, demonstrating that combined modalities can only be used if appropriate selection of response and separation of signals is possible. Whilst building a monolithic network to process combined modality vectors may not seem intuitive, this is one approach to using multiple modalities of information for classification. Our multi-net approach is an alternative that seems to offer benefit. 


\subsection{Classifying Number Magnitude and Articulation}

The combination of modalities to improve classification is a subject that has relevance to developmental psychology. For example, the study of mathematical abilities has concentrated on the way different numerical modalities are processed [4], [13], [14]. Dehaene's triple code model of numerical abilities includes processing of Arabic and spoken numbers, together with an internalised magnitude representation of number [4]. Such an internal representation of number can be obtained through a process of subitization or estimation [9]. Of specific interest here is the way in which the internal magnitude representation of number interacts with number articulation. We simulate the linkage of the internal representation of number and its phonetic representation [1], [3].

We use number magnitude as the primary vector, using a 1-dimensional SOM with 66 neurons as a number line. Input to the SOM is formed as a 66-dimensional binary vector where each magnitude is identified with a block of three digits. The collateral vector is formed as a number articulation, with a SOM consisting of 16 by 16 neurons, showing the representation between the spoken form of the numbers one to twentytwo. For example, the numbers seven, seventeen and eleven all have similar sounds, and are hence clustered together within the SOM. Input to the SOM is formed as a 16-dimensional vector representing the phonemes used in the number word, with value taken as the order found within the Collins English Dictionary and Thesaurus [18], with zero representing the absence of a phoneme for shorter number words.

The training and testing data sets where selected randomly from the numbers 1 to 22 , with 16 numbers in the training set and 6 in the test set $(2,3,10,14,15,19)$. The entire system was trained for 1000 epochs on the training data, with initial random weights, Gaussian neighbourhood function with initial radius for the primary map as 33 , and the collateral 8 , reducing to 1 , and exponential learning rate starting at 0.5 . The Hebbian weight connections were normalised and a learning rate of 0.5 was used.

Once trained, the system was tested on both the magnitude and phonetic forms of all 22 numbers. Looking at the magnitude SOM in isolation, the system correctly ordered all 22 magnitudes such that 1 was at one end of the map and 22 at the other, with all intermediate values in ascending order, including those that were not in the training set. This demonstrates the SOMs ability to generalise to new patterns based upon their apparent magnitude. However, the testing of the phonetic SOM shows the inability to correctly classify $2,3,10,14,15$ and 19 (those not in the training set). The SOM is able to associate these values with those based upon their phonetic similarity, for example 2 is classified as 1, however the SOM cannot distinguish between values, attempting to provide an existing specific label rather than a distinct value.

To determine if the combined system can improve upon this classification, the six misclassified values were presented to the magnitude SOM, and its output used to activate the Hebbian link to produce a corresponding articulation output. The corresponding Hebbian output provides new associations for the six values. Here 14, 15 and 19 are now distinct whilst being associated with the 'teens' cluster, improving classification by distinguishing these values whilst retaining their similarity to their phonetic counterparts. However, 2, 3 and 10 are associated with 20,12 and 21, respectively, a worse classification than previously achieved. The improvement in classification is marginal, having both beneficial and detrimental affects. However, the 
combined system has the advantage that we can improve classification with another modality of information.

To understand if this can be achieved in a monolithic solution rather than the multi-net we describe, we trained a single SOM on a combined set of input modalities. Here, both the magnitude and phonetic representations for the training and testing sets described above where combined into appropriate 82-dimensional vectors. A single SOM consisting of 16 by 16 units was trained using the same parameters as above for 1000 epochs, with an initial neighbourhood radius of 8 units. The results show a map that demonstrates the relationship between the phonetic representations alone, with no magnitude information portrayed. The responses for the six test values gives a similar level of classification as that for the single phonetic SOM described above, with all six values being associated with those that are phonetically similar. However, the loss of magnitude information means that there is no understanding of the relationship between the numbers, nor how to translate between a magnitude and an articulation, or vice-versa. This loss of modality information supports the use of split modalities in a combined system, such as we have described.

\section{Conclusion}

We have presented a modular co-operative neural network system that incorporates unsupervised networks, of the same architecture and topology, that learn to classify a set of patterns based on partial information, either primary information or collateral information, about the patterns. The combiner, a Hebbian network, learns to associate not only the two winning units on the primary and collateral maps, but between the respective neighbourhoods of the two winners.

Note that the number of units in our networks is smaller than, say, in Sharkey's supervised multi-net simulations. We have been parsimonious perhaps compensating for manually assigning the classifiers to the primary or the collateral sub-vectors. However, this manual assignment is offset partially by the fact that the two classifying networks have exactly the same topology and aspects of the training, including regimen, that is the way the neighbourhood distances and learning rates are changed, for the two networks are exactly the same. The combiner in our system shares the same learning paradigm, unsupervised learning, and indeed the SOM architecture is a variant of the Hebbian architecture [20].

A comparison between our modular system and that of a unity network demonstrates improvement. In classifying images and text the unity network does not demonstrate any linkage between images and text, whereas the modular system allow the neighbourhood of images to be associated with neighbourhoods of text. For magnitudes and articulated numerical labels, a unity network provides a comparable classification to that of just the phonetic representations, demonstrating that the phonetic information dominates the magnitude information. 


\section{Acknowledgements}

The authors acknowledge the support of the EPSRC sponsored Scene of Crime Information System project (Grant No.GR/M89041) jointly undertaken by the Universities of Sheffield and Surrey and supported by five police forces in the UK. The authors would also like to thank the UK Police Training College at Hendon for supplying the scene-of-crime images and $\mathrm{Mr} \mathrm{C}$. Handy for transcribing the image collateral text. Lastly, the authors would like to thank the anonymous reviewers of this paper for their constructive comments.

\section{References}

[1] Ahmad, K., Casey, M. \& Bale, T. (2002). Connectionist Simulation of Quantification Skills. Connection Science, vol. 14(3), pp. 165-201.

[2] Ahmad, K., Vrusias, B. \& Tariq, M. (2002). Co-operative Neural Networks and 'Integrated' Classification. Proceedings of the 2002 International Joint Conference on Neural Networks (IJCNN'02), vol.2, pp. 1546-1551.

[3] Bale, T.A. (1998). Modular Connectionist Architectures and the Learning of Quantification Skills. Unpublished doctoral thesis. Guildford, UK: University of Surrey.

[4] Dehaene, S. (1992). Varieties of Numerical Abilities. In Numerical Cognition (1993), pp.1-42. Cambridge, MA.: Blackwell Publishers.

[5] Haralick, R.M., Shanmugam, K. \& Dinstein, I. (1973). Textural Features for Image Classification. IEEE Transactions on Systems, Man, and Cybernetics, vol. SMC-3(6), pp.610621.

[6] Hebb, D.O. (1949). The Organization of Behavior: A Neuropsychological Theory. New York: John Wiley \& Sons.

[7] Jacobs, R.A., Jordan, M.I., \& Barto, A.G. (1991). Task Decomposition through Competition in a Modular Connectionist Architecture: The What and Where Vision Tasks. Cognitive Science, vol. 15, pp. 219-250.

[8] Jing, X., \& Zhang, D. (2003) Face recognition based on linear classifiers combination. Neurocomputing, vol. 50, pp. 485-488.

[9] Kaufman, E.L., Lord, M.W., Reese, T.W. \& Volkmann, J. (1949). The Discrimination of Visual Number. American Journal of Psychology, vol. 62, pp. 498-525.

[10] Kittler, J., Hatef, M. Duin, R.P.W. \& Matas, J. (1998). On Combining Classifiers. IEEE Transactions on Pattern Analysis and Machine Intelligence, vol. 20(3), pp. 226-239.

[11] Kohonen, T. (1997). Self-Organizing Maps, 2nd Ed. Berlin, Heidelberg, New York: Springer-Verlag.

[12] Lawrence, S., Giles, C.L., Ah Chung Tsoi \& Back, A.D. (1997). Face Recognition: A Convolutional Neural Network Approach. IEEE Transactions on Neural Networks, vol. 8 (1) pp 98-113.

[13] McCloskey, M. (1992). Cognitive Mechanisms in Number Processing and Calculation: Evidence from Dyscalculia. In Dehaene, S. (Ed), Numerical Cognition (1993), pp. 107-157. Cambridge, MA.: Blackwell Publishers.

[14] McCloskey, M., Caramazza, A. \& Basili, A. (1985). Cognitive Mechanisms in Number Processing and Calculation: Evidence from Dyscalculia. Brain and Cognition, vol. 4, pp. 171-196.

[15] Schettini, R., Brambilla, C. \& Cusano, C. (2002). Content-based Classification of Digital Photos. In Roli, F. \& Kittler, J. (Eds), Proceedings of the Third International Workshop on Multiple Classifier Systems (MCS 2002), LNCS 2364, pp. 272-282. 
[16] Sharkey, A.J.C. (1999). Multi-net Systems. In Sharkey, A.J.C. (Ed), Combining Artificial Neural Nets: Ensemble and Modular Multi-Net Systems, pp.1-30. Berlin, Heidelberg, New York: Springer-Verlag.

[17] Sharkey, A.J.C. (2002). Types of Multinet System. In Roli, F. \& Kittler, J. (Eds), Proceedings of the Third International Workshop on Multiple Classifier Systems (MCS 2002), LNCS 2364, pp. 108-117.

[18] Sinclair, J. M. (Ed.) (1993) Collins English Dictionary and Thesaurus. Harper Collins.

[19] Srihari R.K., (1995). Use of Collateral Text in Understanding Photos, Artificial Intelligence Review, special issue on Integrating Language and Vision, Volume 8, pp. 409-430.

[20] Willshaw, D.J. \& von der Malsburg, C. (1976). How Patterned Neural Connections can be set up by Self-Organization. Proceedings of the Royal Society, Series B, vol. 194, pp. 431445.

[21] Zhou, X.S., Rui Y. \& Huang S.T. (1999). Water-filling: A Novel Way for Image Structural Feature Extraction. Proceedings of the IEEE International Conference on Image Processing, pp. 570-574. 